ARTICLE OPEN

Check for updates

\title{
Proteomics of REPLICANT perfusate detects changes in the metastatic lymph node microenvironment
}

Julia Stevenson ${ }^{1}$, Rachel Barrow-McGee ${ }^{1}$, Lu Yu (D) ${ }^{2}$, Angela Paul $^{2}$, David Mansfield ${ }^{3}$, Julie Owen ${ }^{4}$, Natalie Woodman ${ }^{4}$, Rachael Natrajan (iD) ${ }^{1}$, Syed Haider ${ }^{1}$, Cheryl Gillett ${ }^{4}$, Andrew Tutt ${ }^{1}$, Sarah E. Pinder (D) $^{5}$, Jyoti Choudary ${ }^{2}$ and Kalnisha Naidoo (D) $^{1,6 凶}$

In breast cancer (BC), detecting low volumes of axillary lymph node (ALN) metastasis pre-operatively is difficult and novel biomarkers are needed. We recently showed that patient-derived ALNs can be sustained ex-vivo using normothermic perfusion. We now compare reactive (tumour-free; $n=5$ ) and macrometastatic (containing tumour deposits $>2 \mathrm{~mm} ; n=4$ ) ALNs by combining whole section multiplex immunofluorescence with TMT-labelled LC-MS/MS of the circulating perfusate. Macrometastases contained significantly fewer B cells and T cells (CD4 ${ }^{+} / \mathrm{CD} 8^{+} /$regulatory) than reactive nodes $(p=0.02)$. Similarly, pathway analysis of the perfusate proteome (119/1453 proteins significantly differentially expressed) showed that immune function was diminished in macrometastases in favour of 'extracellular matrix degradation'; only 'neutrophil degranulation' was preserved. Qualitative comparison of the perfusate proteome to that of node-positive pancreatic and prostatic adenocarcinoma also highlighted 'neutrophil degranulation' as a contributing factor to nodal metastasis. Thus, metastasis-induced changes in the REPLICANT perfusate proteome are detectable, and could facilitate biomarker discovery.

npj Breast Cancer (2021)7:24; https://doi.org/10.1038/s41523-021-00227-7

\section{INTRODUCTION}

Precise histopathological quantification of axillary tumour burden remains central to the management of patients with early-stage breast cancer $(\mathrm{BC})^{1}$. This volumetric assessment of metastatic nodal disease is determined by counting the total number of metastatic axillary lymph nodes (ALNs), and measuring the size of the largest tumour deposit ${ }^{1}$. Recent efforts to delineate when it is safe to leave metastatic ALNs in situ, obviating the risks associated with a surgical ALN dissection (ALND), have proved controversial ${ }^{1-5}$. At a biological level, we have yet to determine at which size/ volume a tumour deposit 'switches off' the immune response in an ALN, facilitating tumour growth and spread. In other words, how tumour biology and intra-tumour heterogeneity (ITH) affect ALN colonisation is unclear ${ }^{6}$.

This uncertainty is confounded by the fact that while preoperative imaging can reliably quantify high volumes of axillary disease, detecting smaller amounts is difficult, even when coupled with a needle-biopsy ${ }^{7}$. Furthermore, no reliable predictive biomarkers of axillary tumour burden exist at present. This is partly because every surgically excised ALN has to be formalin-fixed and paraffin-wax embedded (FFPE) for diagnosis and treatment planning ${ }^{1,8}$. As such, obtaining human nodal tissue for biomarker discovery and validation is difficult.

Although the liquid biopsy overcomes these issues of access and sample quantity, the complexity of blood has proved challenging for biomarker discovery ${ }^{9}$. Exactly how much nodal disease is required for circulating tumour DNA (ctDNA) to become detectable has yet to be determined. Interestingly, it has been shown that ctDNA can be identified pre-operatively in treatment naïve patients with more than three metastatic ALNs, and that levels drop following ALN removal ${ }^{10}$. However, since cell death or 'shedding' is required to release ctDNA, it could be argued that 'non-shedding' tumours might escape detection by this method altogether ${ }^{11}$. For these reasons, monitoring ctDNA in early-stage cancer is not recommended currently ${ }^{11}$.

With regard to proteomics, there is little concordance between the few studies that have tried to identify biomarkers of axillary disease in human serum/plasma samples ${ }^{12-17}$. Similarly, human tissue studies comparing the proteomes of primary $\mathrm{BC}$ to matched ALN metastases have yielded disparate data ${ }^{18-23}$. On the whole, ALN metastases have been shown to be similar to the primary BC at a protein level but minimal overlap was seen between studies. No studies thus far have compared the protein expression of reactive to metastatic ALN tissue.

Intriguingly, lymph seems to contain higher concentrations of circulating biomarkers, particularly in the early stages of metastasis $^{24}$. Proteomic studies have shown that lymph reflects the pathophysiology of the tissue from which it derives ${ }^{25-27}$, and has recently been shown to be relevant to melanoma biomarker discovery and stage prediction ${ }^{24}$. To our knowledge, no such studies have been performed on lymphatic exudate from BC patients undergoing an ALND however.

In the 'REPLICANT' study, we recently showed that human ALNs from BC patients can be sustained ex vivo for scientific investigation using normothermic perfusion ${ }^{8}$. Herein, we characterise the proteome of the circulating fluid collected from these perfused ALNs ('perfusate') using Tandem Mass Tag (TMT) labelled mass spectrometry (MS)-based shotgun proteomics, and show that it can discriminate between reactive (tumour-free) and macrometastatic ALNs (i.e. containing a tumour deposit $>2 \mathrm{~mm}$ in maximal dimension).

\footnotetext{
${ }^{1}$ The Breast Cancer Now Toby Robins Research Centre, The Institute of Cancer Research, London, UK. ${ }^{2}$ Division of Cancer Biology, The Institute of Cancer Research, London, UK. ${ }^{3}$ Division of Radiotherapy and Imaging, The Institute of Cancer Research, London, UK. 'King's Health Partners Cancer Biobank, Guy's Comprehensive Cancer Centre, London, UK. ${ }^{5}$ School of Cancer and Pharmaceutical Sciences, King's College London, Guy's Comprehensive Cancer Centre, London, UK. ${ }^{6}$ Department of Cellular Pathology, Imperial College Healthcare NHS Trust, Charing Cross Hospital, London, UK. ${ }^{\bowtie}$ email: kalnisha.naidoo1@nhs.net
} 


\section{RESULTS}

Immune cells decrease significantly as tumour grows within an ALN

All of the ALNs collected during the study (10 perfused and 10 baseline control) underwent multiplex immunofluorescence (MIF). Representative images of a reactive (Fig. 1A) and a macrometastatic ALN (Fig. 1B) are shown ( $\times 20$ magnification; field of view: $670 \mu \mathrm{m} \times 500 \mu \mathrm{m}$ ). The first panels (left to right) show MIF staining, while second panels show the cell phenotype maps that were generated algorithmically from the MIF images. In addition to analysing cell density (i.e. total cells per $\mathrm{mm}^{2}$ ) over the whole tissue section, the tissue in metastatic nodes was segmented (third panel in Fig. 1B) into areas containing mostly cancer cells ('tumour'; red), stromal regions ('stroma'; light grey), and areas comprising mainly lymphoid cells ('lymphoid'; green). The node containing a micrometastasis (i.e. a tumour deposit measuring $0.2-2 \mathrm{~mm}$ ) was excluded from further analysis (including proteomics) since the tumour cells had cut out after sectioning, thus confounding evaluation. Thus, nine ALNs were analysed in total (i.e. five reactive and four macrometastatic).

Although some quantitative differences in immune composition were seen between control and perfused ALNs, none reached
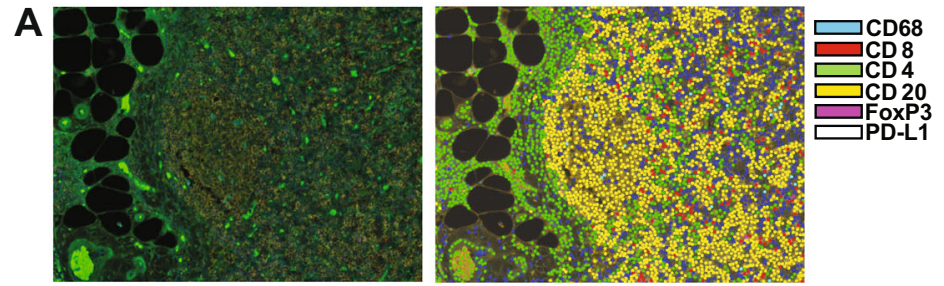

B
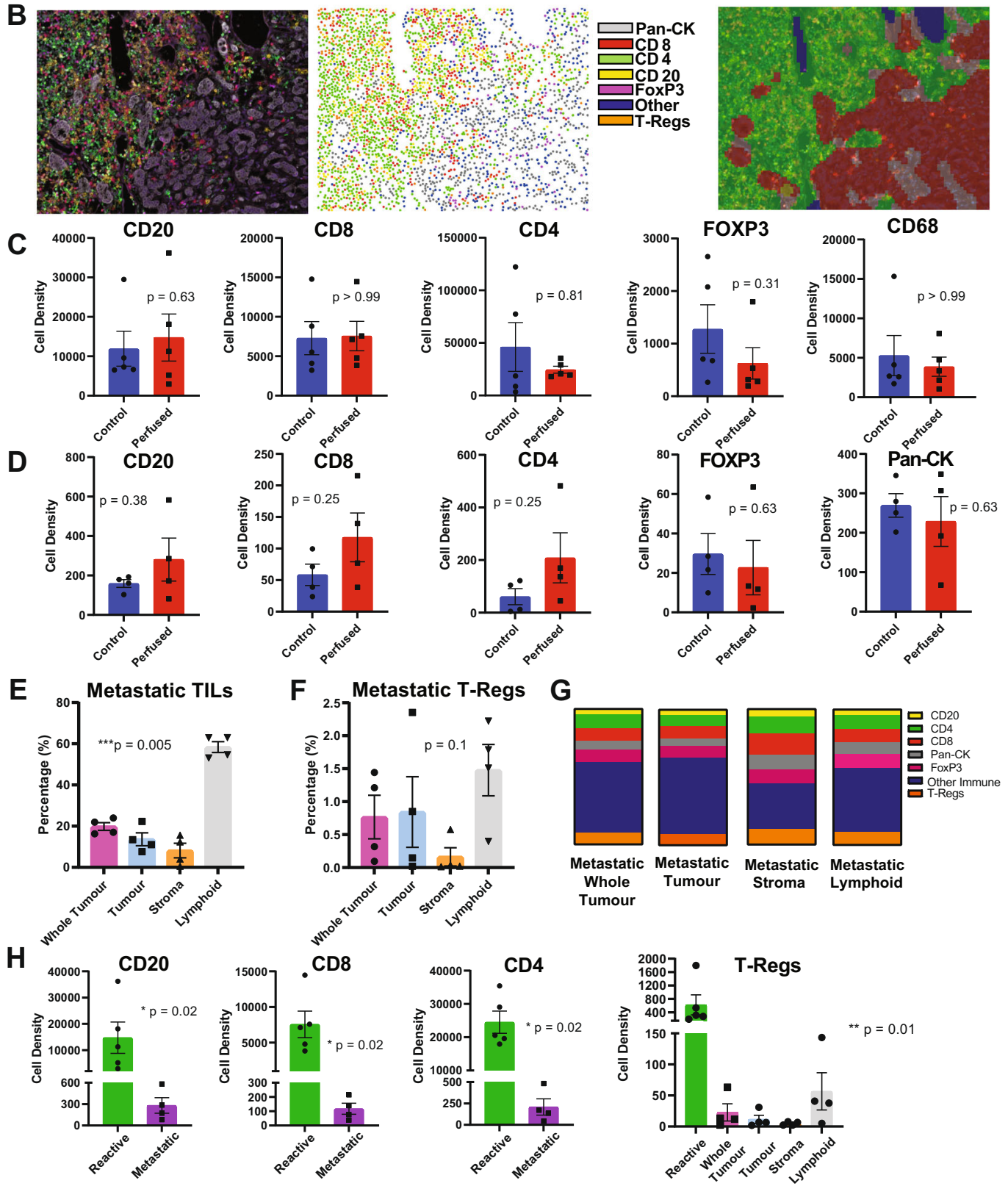
Fig. 1 Multiplex immunofluorescence of REPLICANT axillary lymph nodes (ALNs). Reactive $(n=5)$ and macrometastatic $(n=4)$ ALNs were fluorescently stained for CD8, CD4, CD20, FoxP3 and PD-L1; reactive nodes, for CD68 in addition; metastatic nodes, additionally for pancytokeratin (pan-CK). Representative images of a reactive (A) and macrometastatic (B) node are shown (x20 magnification; field of view: $670 \mu \mathrm{m} \times 500 \mu \mathrm{m})$. From left to right, the first panels show immunofluorescent staining; second panels show cell phenotype maps, which were generated algorithmically. In metastatic nodes, the tissue also was segmented (third panel in B) into areas containing mostly cancer cells ('tumour'; red), stromal regions ('stroma'; light grey), and areas comprising mainly lymphoid cells ('lymphoid'; green) for tumour infiltrating lymphocyte (mTILs) and PD-L1 analysis. No statistically significant differences in immune composition were seen between control (fixed at baseline) and perfused nodes (reactive nodes are shown in $\mathbf{C}$, metastatic in $\mathbf{D}$; cell density = total cells $/ \mathrm{mm}^{2}$; Wilcoxon test). Subsequent analysis was therefore done on perfused ALNs only. The percentage of mTILs across the whole tissue section of metastatic ALNs ('whole tumour'), as well as in 'tumour', 'stroma', and 'lymphoid' areas, is shown in $\mathbf{E}(n=4)$. The differences between these regions was statistically significant ( $p<0.0001$; Kruskal-Wallis test). The regional distribution of regulatory T cells (T-regs) did not differ significantly however (F). The co-localisation of PD-L1 (average signal intensity) with other cell markers is shown in G. 49\% (whole tumour) and 30-54\% (regional) of the PDL1 staining did not co-localise with any of the other markers that we had stained for ('other immune'). Most of the remaining signal was present on T-lymphocytes (36\% (whole tumour); $32 \%$ (tumour); $46 \%$ (stroma); and 37\% (lymphoid)), with cancer cells contributing between 5 and $10 \%$, and B cells $<5 \%$, to overall intensity. $\mathbf{H}$ The average cell density of CD20 ${ }^{+} \mathrm{B}$ cells $(p=0.02), \mathrm{CD} 8^{+} \mathrm{T}$ cells $(p=0.02), \mathrm{CD} 4{ }^{+} \mathrm{T}$ cells $(p=$ 0.02 ) and T-regs $(p=0.02)$ was significantly decreased in nodes replaced by macrometastases (latter, Kruskal-Wallis; former three, Mann-Whitney). (Graphs show mean with standard error of the mean (SEM)).

statistical significance (reactive nodes $(n=5)$ are shown in Fig. $1 \mathrm{C}$ and macrometastatic nodes $(n=4)$ are shown in Fig. 1D). These data provide further evidence that perfusion does not appear to alter the tumour-immune microenvironment within $\mathrm{ALNs}^{8}$, and therefore all subsequent analysis was done on perfused ALNs only.

We calculated the percentage of tumour infiltrating lymphocytes in the metastatic tumour (mTILs; Fig. 1E) ${ }^{28}$, in the whole section and in the three regions described above. 'Lymphoid' areas, unsurprisingly, had the highest concentration of mTILs, while the lowest concentration was seen within 'tumour' foci. Importantly, the difference in mTILs between the compartments was statistically significant $(p<0.0001)$, suggesting that stromal mTILs might not be a surrogate for whole tumour mTILs in metastatic nodes. Regulatory T cells (T-regs) are an immunosuppressive subset of $\mathrm{CD}^{+} \mathrm{T}$ cells which also express FOXP3 ${ }^{29}$. These cells can dampen the anticancer immune response, promoting tumour growth and progression; they also express immunecheckpoint molecules and, as such, are the target of immune checkpoint inhibitor therapies ${ }^{29}$. We therefore evaluated their distribution in macrometastatic ALNs. T-regs distribution followed the pattern of total $\mathrm{mTIL}$ distribution in the various compartments, but did not reach statistical significance (Fig. 1F).

The ubiquitous co-expression of PD-L1 made cell density quantification difficult. To overcome this, we calculated the average signal intensity for each cell type within each macrometastatic node (Fig. 1G). As can be seen, 49\% (whole tumour) and $30-54 \%$ (regional) of the PD-L1 staining did not co-localise with any of the other markers that we had stained for; these are probably macrophages or dendritic cells ${ }^{30}$. Most of the remaining signal was present on T-lymphocytes (36\% (whole section) and $32-46 \%$ (regional)), with cancer cells contributing between 5 and $10 \%$, and $\mathrm{B}$ cells $<5 \%$, to overall intensity. Furthermore, the signal distribution was consistent in each area quantified (Representative images of PD-L1 MIF are shown in Supplementary Fig. 1).

Finally, we compared the immune composition of reactive to metastatic ALNs (Fig. 1H). The average cell density of B cells ( $p=$ $0.02)$, cytotoxic T cells $(p=0.02)$; T-helper cells $(p=0.02)$ and Tregs $(p=0.02)$ decreased significantly when macrometastases were present. This reflects the fact that nodal architecture is destroyed and replaced by metastatic tumour during ALN colonisation ${ }^{6}$.

\section{The perfusate proteome reflects the pathophysiology of perfused ALNs}

Proteomic analysis of the perfusate samples $(n=9)$ identified 1453 proteins in total (Supplementary Table 1). Of these, 119 (8\%) were significantly differentially expressed (DE) between reactive and metastatic samples (Table $1 ; p \leq 0.05$ ). Hierarchical cluster analysis of these 119 significantly differentially expressed proteins
(DEP; Fig. 2) showed a clear separation of reactive and metastatic samples. This suggests that certain biological features within metastatic nodes are not only distinctive, but shared across different patients.

The 57 significantly upregulated proteins in reactive ALNs $(n=5)$ and the 62 significantly up-regulated proteins in metastatic ALNs $(n=4)$ were subjected to pathway analysis in ConsensusPathDB ${ }^{31}$. The 10 most enriched pathways for both disease states are shown in Fig. 3A (reactive) and B (metastatic).

In reactive nodes, as expected, the 10 most common pathways identified all linked to immunity, both innate and adaptive. Conversely, in the metastatic perfusate samples, proteins reflecting active immune function were poorly represented, supporting the picture painted by MIF. Most of the pathways identified in these nodes relate to extracellular matrix (ECM) degradation. The ECM is known to regulate cell behaviour and differentiation in lymph nodes (LNs) in both health and disease, changing dynamically in response to injury ${ }^{32-36}$. The fact that 'keratinisation' was identified in the metastatic perfusate samples reflects the presence of tumour cells within the ALNs, since only epithelial cells contain keratin. These cells are not a normal LN constituent, and thus served as a good positive control. The only immune process identified in the metastatic perfusate samples was 'neutrophil degranulation'.

Since 'neutrophil degranulation' had been identified as being significantly up-regulated in metastatic perfusate samples, we reviewed the haematoxylin and eosin (H\&E) sections of the nine perfused $\mathrm{ALNs}^{8}$ to see if the absolute number of neutrophils was increased in metastatic nodes (Fig. 3C). Although there was a trend towards higher numbers of neutrophils in metastatic nodes, this did not reach statistical significance $(p=0.19)$.

Overall, these data suggest that the proteome reflects the pathophysiology of the perfused ALNs, and that a shift to 'ECM degradation' and 'neutrophil degranulation' can be used to infer when macrometastases are present or not.

\section{Analysing differences between reactive and metastatic perfusate samples reveals novel patterns of protein dysregulation}

In order to see how the perfusate proteome compared to that of primary BC tissue samples, we mined TCGA samples which underwent proteomic analysis in the CPTAC study ${ }^{37-39}$. Qualitatively, 1361 of the 1453 perfusate proteins were present in the TCGA(CPTAC) dataset (i.e. 94\% overlap; data not shown). However, since an average of 11,632 proteins/tumour were found in that study, the perfusate probably contains a fraction of the proteins expressed in primary $\mathrm{BC}^{39}$.

We then stratified the TCGA(CPTAC) samples into those from patients who had undergone a sentinel LN biopsy (SLNB) only 
Table 1. 119 significantly differentially expressed proteins between reactive $(n=5)$ and metastatic $(n=4)$ perfusate.

\begin{tabular}{|c|c|c|c|c|c|c|}
\hline Uniprot ID & Description & $\begin{array}{l}\text { \# Unique } \\
\text { peptides }\end{array}$ & Reactive $_{\text {AVE }}$ & Metastatic $_{\mathrm{AVE}}$ & log2ratio & $T$-test \\
\hline P02655 & Apolipoprotein C-II & 5 & 153.72 & 32.83 & -2.23 & 0.03 \\
\hline P00568 & Adenylate kinase isoenzyme 1 & 8 & 141.80 & 47.70 & -1.57 & 0.04 \\
\hline P68036 & Ubiquitin-conjugating enzyme E2 L3 & 2 & 141.16 & 48.55 & -1.54 & 0.01 \\
\hline A1LOTO & Acetolactate synthase-like protein & 2 & 141.06 & 48.73 & -1.53 & 0.01 \\
\hline P51888 & Prolargin & 5 & 139.20 & 50.98 & -1.45 & 0.005 \\
\hline P02656 & Apolipoprotein C-III & 2 & 139.16 & 51.00 & -1.45 & 0.03 \\
\hline P35611 & Alpha-adducin & 11 & 138.52 & 51.85 & -1.42 & 0.04 \\
\hline P40227 & T-complex protein 1 subunit zeta & 5 & 137.98 & 52.58 & -1.39 & 0.05 \\
\hline O00159 & Unconventional myosin-lc & 13 & 136.70 & 54.08 & -1.34 & 0.03 \\
\hline O75955 & Flotillin-1 & 4 & 135.58 & 55.58 & -1.29 & 0.04 \\
\hline P35232 & Prohibitin & 2 & 159.43 & 65.55 & -1.28 & 0.004 \\
\hline P50990 & T-complex protein 1 subunit theta & 11 & 134.98 & 56.30 & -1.26 & 0.03 \\
\hline P52943 & Cysteine-rich protein 2 & 3 & 134.94 & 56.38 & -1.26 & 0.03 \\
\hline P48643 & T-complex protein 1 subunit epsilon & 9 & 134.48 & 56.88 & -1.24 & 0.01 \\
\hline Q8WUM4 & Programmed cell death 6-interacting protein & 9 & 134.40 & 56.98 & -1.24 & 0.01 \\
\hline 000151 & PDZ and LIM domain protein 1 & 8 & 134.34 & 57.08 & -1.23 & 0.03 \\
\hline P17987 & T-complex protein 1 subunit alpha & 11 & 134.24 & 57.20 & -1.23 & 0.03 \\
\hline O75947 & ATP synthase subunit $d$, mitochondrial & 3 & 133.98 & 57.53 & -1.22 & 0.04 \\
\hline Q9NQ79 & Cartilage acidic protein 1 & 3 & 133.82 & 57.73 & -1.21 & 0.03 \\
\hline P35998 & $26 S$ proteasome regulatory subunit 7 & 4 & 130.98 & 61.25 & -1.10 & 0.02 \\
\hline O75915 & PRA1 family protein 3 & 4 & 130.18 & 62.33 & -1.06 & 0.04 \\
\hline O95747 & Serine/threonine-protein kinase OSR1 & 5 & 130.04 & 62.43 & -1.06 & 0.04 \\
\hline P28289 & Tropomodulin-1 & 10 & 130.02 & 62.48 & -1.06 & 0.03 \\
\hline Q969G5 & Caveolae-associated protein 3 & 5 & 129.74 & 62.83 & -1.05 & 0.05 \\
\hline P16152 & Carbonyl reductase [NADPH] 1 & 5 & 129.52 & 63.08 & -1.04 & 0.05 \\
\hline Q9UNH7 & Sorting nexin- 6 & 2 & 129.18 & 63.53 & -1.02 & 0.04 \\
\hline P11171 & Protein 4.1 & 19 & 129.16 & 63.53 & -1.02 & 0.05 \\
\hline P30626 & Sorcin & 7 & 128.06 & 64.95 & -0.98 & 0.04 \\
\hline P26447 & Protein S100-A4 & 3 & 127.96 & 65.05 & -0.98 & 0.03 \\
\hline P04083 & Annexin $A 1$ & 15 & 127.46 & 65.68 & -0.96 & 0.04 \\
\hline P48059 & LIM and senescent cell antigen-like-containing domain protein 1 & 3 & 127.00 & 66.25 & -0.94 & 0.02 \\
\hline Q04446 & 1,4-alpha-glucan-branching enzyme & 3 & 125.16 & 68.53 & -0.87 & 0.05 \\
\hline P36969 & Phospholipid hydroperoxide glutathione peroxidase & 2 & 124.38 & 69.50 & -0.84 & 0.05 \\
\hline P35241 & Radixin & 9 & 123.96 & 70.05 & -0.82 & 0.02 \\
\hline P05546 & Heparin cofactor 2 & 10 & 123.84 & 70.20 & -0.82 & 0.05 \\
\hline Q8TAT6 & Nuclear protein localisation protein 4 homologue & 2 & 123.80 & 70.28 & -0.82 & 0.05 \\
\hline Q9H4A3 & Serine/threonine-protein kinase WNK1 & 5 & 122.78 & 71.55 & -0.78 & 0.03 \\
\hline P60660 & Myosin light polypeptide 6 & 7 & 122.56 & 71.83 & -0.77 & 0.04 \\
\hline P08697 & Alpha-2-antiplasmin & 6 & 122.54 & 71.88 & -0.77 & 0.05 \\
\hline
\end{tabular}




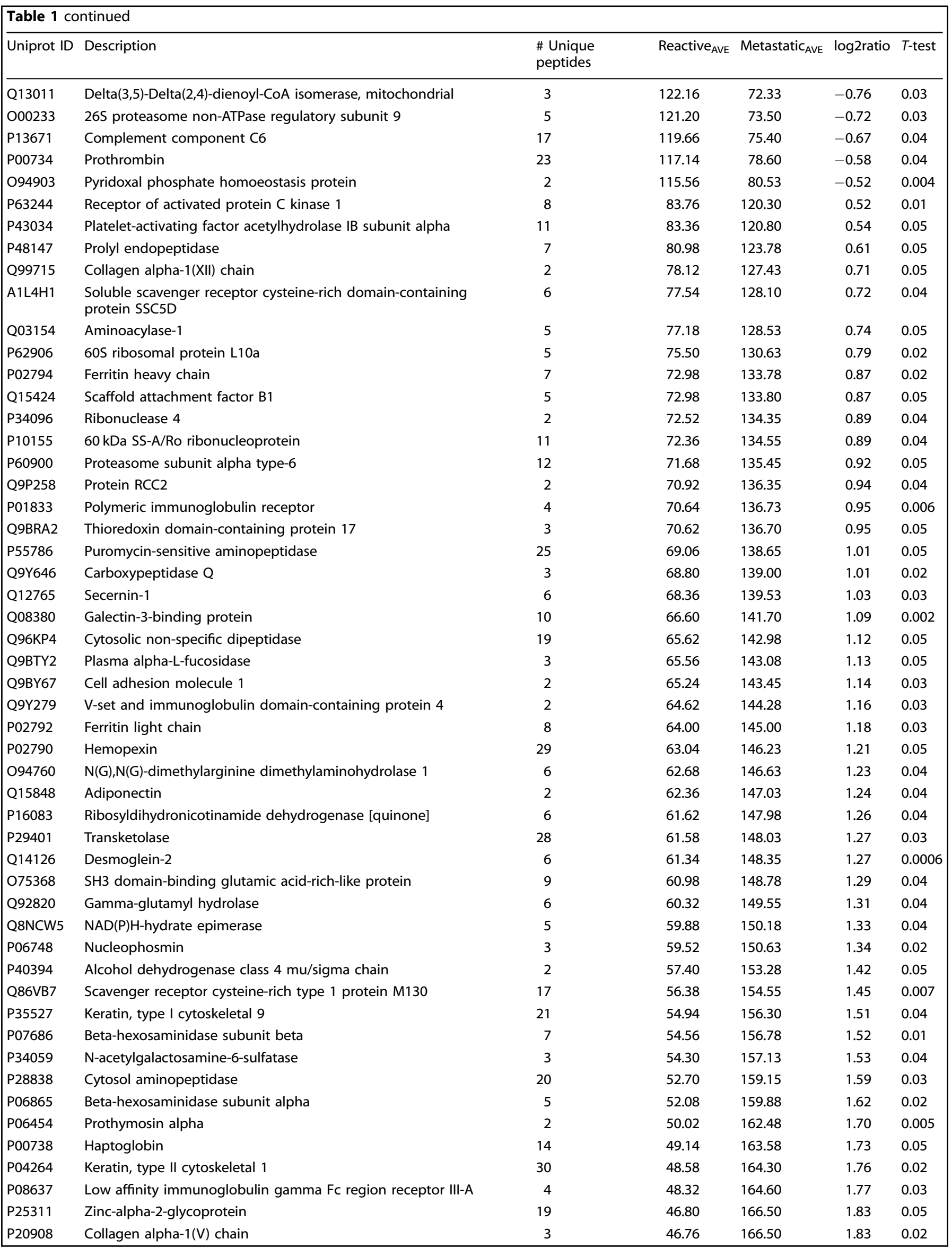




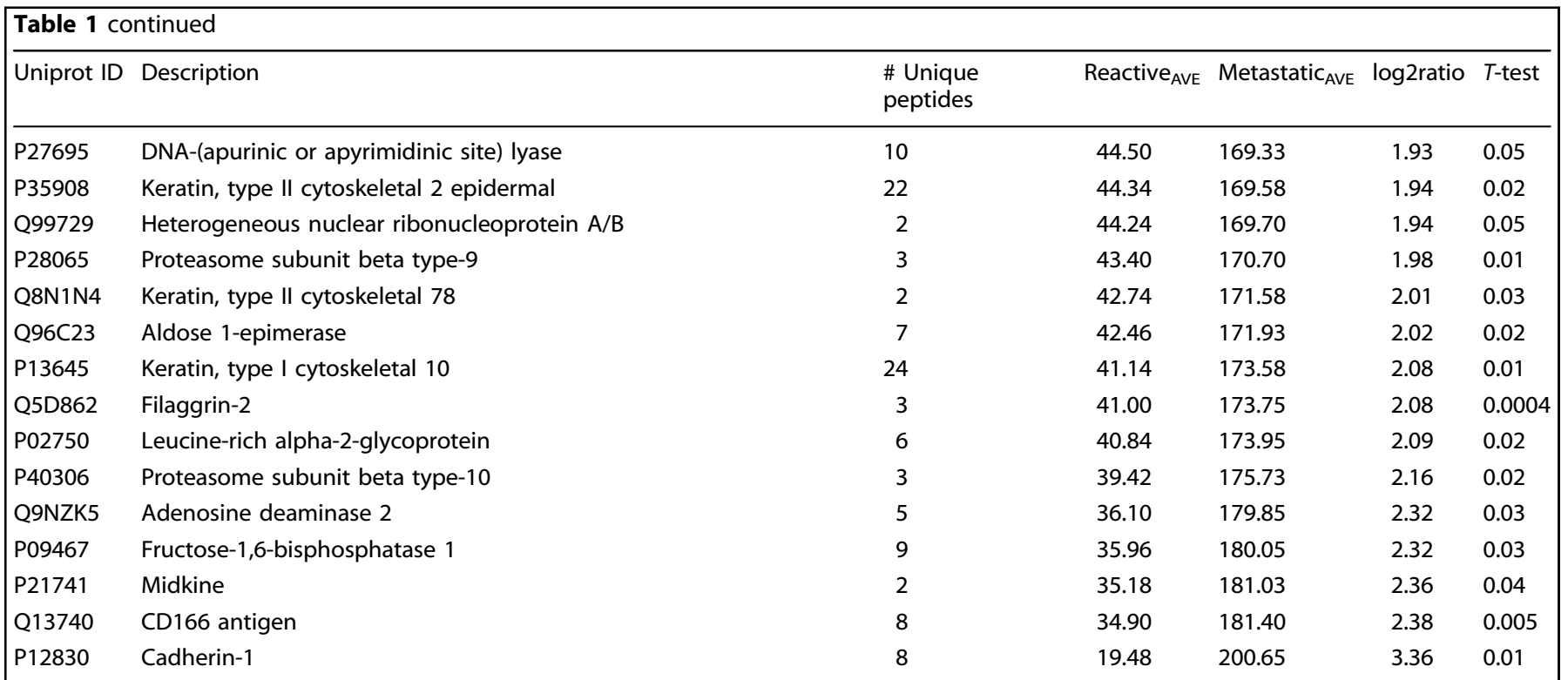

(i.e. no/low axillary tumour burden); those who had also had a completion clearance (CC) (i.e. presumed higher axillary tumour burden than the SLNB group); and those who had had an upfront ALND (i.e. presumed high axillary tumour burden at diagnosis). The rationale for this was that the SLNB group would be similar to the reactive perfusate samples, whilst the CC/ALND groups would be comparable to the metastatic perfusate samples to varying degrees. We tested this hypothesis using the 10 most abundant proteins found in the reactive (namely APOC2, TGM2, AK1, UBE2L3, ILVBL, ASPN, UBE2V1, PRELP, APOC3 and ADD1) and metastatic (namely GALM, KRT10, FLG2, LRG1, PSMB10, ADA2, FBP1, MDK, ALCAM and CDH1) perfusate, respectively.

Eight reactive perfusate proteins were identified in the TCGA (CPTAC) samples, and the DE of three of the proteins between the clinical groups was as expected (Fig. 4A, B). ADD1 was significantly more abundant in patients in the SLNB group $(p=0.01)$. Since phosphoproteomics had been performed in that study, we could also identify which phospho-isoforms of ADD1 were significantly DE between the clinical groups (Fig. 4A); however, the implications of this are currently unknown. ASPN $(p=0.02)$ and PRELP ( $p=$ 0.023 ) were also significantly more abundant in patients in the SLNB group (Fig. 4B). The trends in expression for the other five reactive proteins are shown in Supplementary Fig. $2 \mathrm{~A}$ (post-hoc analysis using Tukey's honestly significantly difference (HSD) test is shown in Supplementary Table 2).

Eight metastatic proteins were identified in the TCGA(CPTAC) samples, but none were significantly $D E$ between the clinical groups (Supplementary Fig. 2B; post-hoc analysis using Tukey's HSD test is shown in Supplementary Table 2). CDH1 expression did increase as axillary tumour burden increased, but this did not reach significance $(p=0.081)$.

Closer inspection of the TCGA(CPTAC) data explained this disparity to a certain extent. Our assumption regarding the 13 patients in this study who had undergone an SLNB alone was correct-none had histopathological evidence of axillary disease following surgery. However, approximately $40 \%$ of the patients in the CC and ALND groups were staged as NO following surgery ${ }^{40}$ i.e. all of the retrieved LNs were reactive, and showed no evidence of metastatic tumour on H\&E staining. Thus, these two groups did not consist entirely of patients with a higher burden of axillary disease, and were not therefore entirely comparable with the metastatic perfusate samples.

The total proteome data for only one study comparing matched primary $B C$ and ALN metastases was available/accessible for comparison $^{19}$. Since this had used a gel-based method of protein separation, fewer proteins (135 in total) were identified; 86 of these were present in the perfusate proteome (64\%; Supplementary Table 3). Quantitative analysis was not feasible for this study.

Comparisons were feasible for two of the above-mentioned BC plasma proteome studies; little overlap was seen between plasma and the perfusate proteome (Fig. 4C). SERPIND1, which was significantly up-regulated in the reactive perfusate samples, was also upregulated in node-negative Her2-positive BC patients ${ }^{17}$. KRT9 was significantly up-regulated in the metastatic perfusate samples; this was found to be up-regulated in node-positive Her2positive patients previously ${ }^{17}$. Lobo et al. found APOC3 to be upregulated in patients with stage I/II BC (i.e. patients with a low axillary tumour burden $)^{16}$; this was significantly up-regulated in the reactive perfusate samples.

Thus, most proteins identified in the perfusate samples have been identified in primary BC tissue, but not in BC plasma, samples previously. Interestingly, the comparison of reactive to metastatic ALN perfusate samples generated different data to that which could be obtained by stratifying primary BC tissue samples according to nodal status.

\section{Neutrophil degranulation is repeatedly highlighted in LN metastasis, across cancers}

To see if certain proteins are conserved during LN metastasis across different carcinomas, we qualitatively compared the perfusate proteome to proteomic studies analysing metastatic LNs from other cancers/sites.

We previously compared primary pancreatic ductal adenocarcinoma (PDAC) and matched LN metastases using laser capture microdissection coupled to multidimensional protein identification technology ${ }^{41}$. Interestingly, 515 of the 854 proteins $(60 \%)$ identified in that study were also present in the perfusate proteome (Supplementary Table 4). The top 10 enriched pathways for these overlapping proteins are shown in Fig. 5A. Once again, 'neutrophil degranulation' was identified as the most enriched pathway between the two datasets.

Muller et al. compared prostate carcinoma tissue from patients with or without LN metastasis using label-free LC/MS/MS ${ }^{42}$. Qualitative comparison of that proteome to the perfusate protein revealed 854 commonly expressed proteins ( $48 \%$ of the 1750 total proteins identified; Supplementary Table 5). The top 10 enriched pathways for these overlapping proteins are shown in Fig. 5B; they 


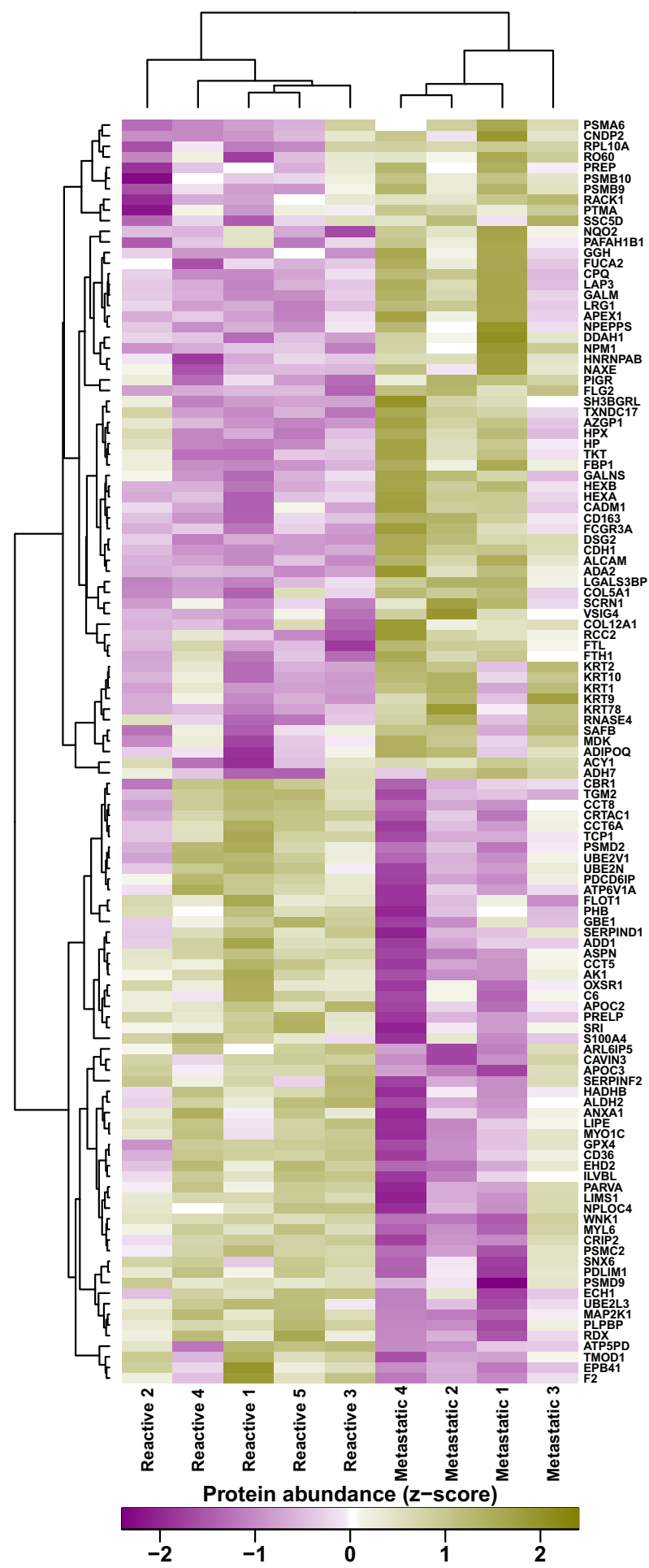

Fig. 2 Hierarchical cluster analysis of the 119 significantly differentially expressed proteins separates reactive from metastatic nodes. A heat map showing hierarchical clustering of the nine perfusate samples (taken from nine different patients). A clear separation of reactive and metastatic nodes is seen.

are remarkably similar to those seen in Fig. 5A, with 'neutrophil degranulation' also being the most enriched pathway in this comparison.

Across the three datasets, 438 common proteins were identified (Supplementary Table 6). Once again, the top 10 enriched pathways (Fig. 6A) were similar to the previous comparisons (Fig. 5A, B). Interestingly, all seven of the 14-3-3 family of proteins; two of the IQGAP family of proteins (IQGAP1 and IQGAP2); four of the 36 human SERPIN proteins (SERPINA1; SERPINA3; SERPINB1 and SERPINH1); four of the S100 proteins (S100A8; S100A9; S100A10 and S100A11); six of the 12 Annexin proteins (ANXA1-6); two of the ezrin/radixin/moesin (ERM) family of proteins (EZN and MSN); three of the 12 aldehyde dehydrogenase protein family (ALDH1A1; ALDH2 and ALDH9A1); and four of the 11 human cathepsins (CSTB, CSTD; CSTG and CSTZ) were conserved across the three cancers. In addition, a number of ECM proteins were conserved across the datasets: 10 collagen subunits; fibronectin, laminin B, periostin, tenascin and vitronectin. Pathway analysis of these 48 conserved proteins is shown in Fig. 6B.

Thus, 'neutrophil degranulation' is recurrently highlighted as the most significant immune pathway in LN metastasis, irrespective of where the primary tumour originates.

\section{DISCUSSION}

We have shown previously that human ALNs can be sustained exvivo for scientific investigation using normothermic perfusion ${ }^{8}$. Using shotgun proteomics, we now show that the protein repertoire of the circulating fluid collected during these experiments ('perfusate proteome') reflects ALN pathophysiology and thus, may be suitable for biomarker discovery.

As expected, MIF analysis of reactive and macrometastatic ALNs confirmed that the total number of $\mathrm{B}$ cells, $\mathrm{CD} 4^{+} \mathrm{T}$ cells, $\mathrm{CD} 8^{+}$ $\mathrm{T}$ cells and T-regs decreases significantly as cancer colonises a node $^{6}$. Interestingly, within macrometastastic nodes, mTIL distribution was significantly higher across the whole ALN section than in areas containing stroma. This result needs to be investigated further in a larger cohort of ALNs. Although TILs are an established biomarker in primary Her-2 positive and triplenegative $B C$, uncertainty exists at present as to precisely how to quantify mTILs in LNs largely for two reasons: first, not all metastases contain stroma; and second, LNs contain a large lymphoid population which confounds assessment ${ }^{28}$. Furthermore, how mTIL infiltration and PD-L1 expression relate to each other in LNs is unclear ${ }^{43,44}$. The ubiquitous co-expression of PD-L1 in our ALNs made cell density quantification difficult. However, by using average staining intensity for each cell type, we were able to show that approximately $30 \%$ of PD-L1 expression localised to T-lymphocytes. Unlike TIL distribution, PD-L1 expression appeared to be uniform in each of the compartments analysed. This may be related to the PD-L1 antibody that we used however ${ }^{45,46}$. Although this clone has been used previously to assess PD-L1 expression in primary and metastatic $B C^{47}$, it is not used routinely for diagnosis. Only the SP142 clone has been approved for diagnostic use ${ }^{48}$, and only in triple-negative BC (not predominant in our cohort). Intriguingly, a positive PD-L1 result using this clone is defined by any staining intensity in immune cells in $>1 \%$ of the tumour. It will be interesting to evaluate this clone in MIF sections of ALNs containing the various subtypes of $B C$ in future experiments, especially in response to immune checkpoint inhibitor $(\mathrm{ICl})$ therapies ${ }^{8}$.

Pathway analysis of the significantly upregulated proteins in reactive and/or metastatic nodes reflected the change in cell composition highlighted by MIF. Reactive nodes showed a maintenance of immune function, whereas macrometastatic nodes showed a loss in immune function (except for 'neutrophil degranulation'), and a shift to ECM degradation and keratinisation. The latter is consistent with the presence of epithelial cells within the ALN. Alterations in the ECM are known to affect fluid flow; lymphangiogenesis; angiogenesis; cancer cell adhesion, migration and invasion; cytokine signalling; and immune modulation ${ }^{49-51}$, all of which contribute to metastasis and colonisation ${ }^{6}$. Unlike the lung, liver or bone however, changes in the ECM at a protein level 
A

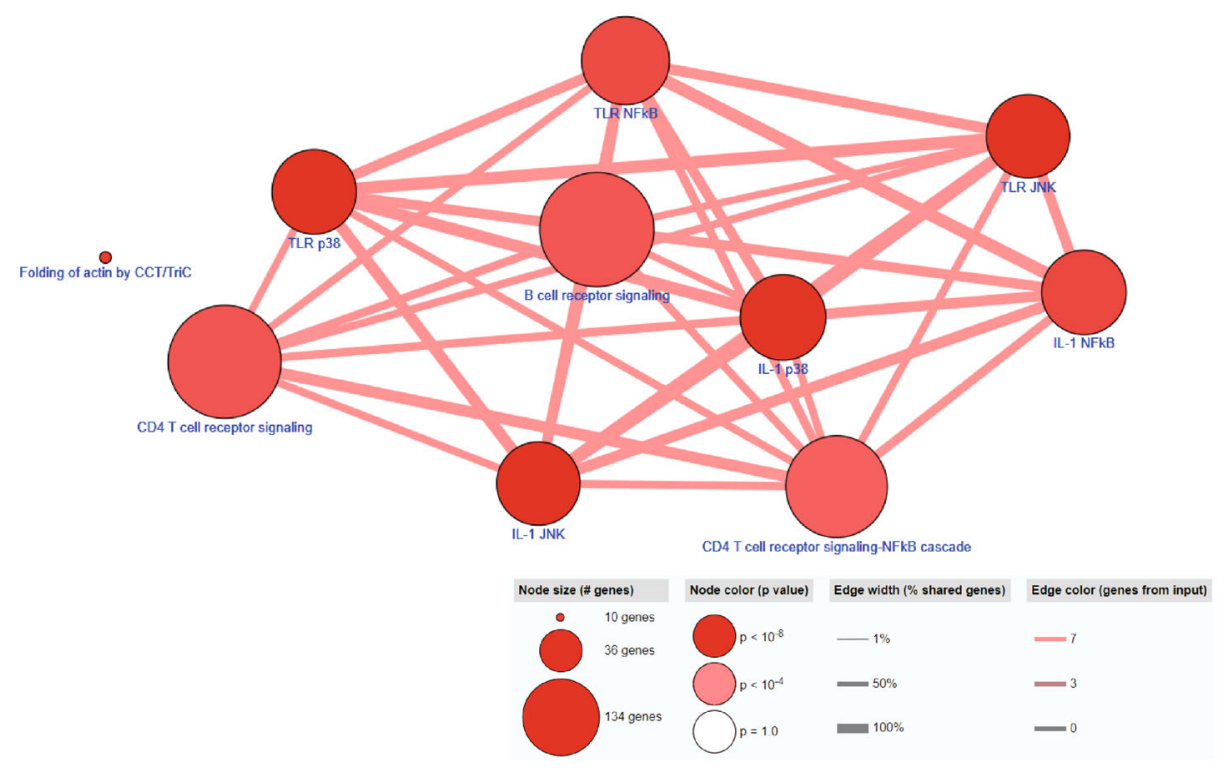

B
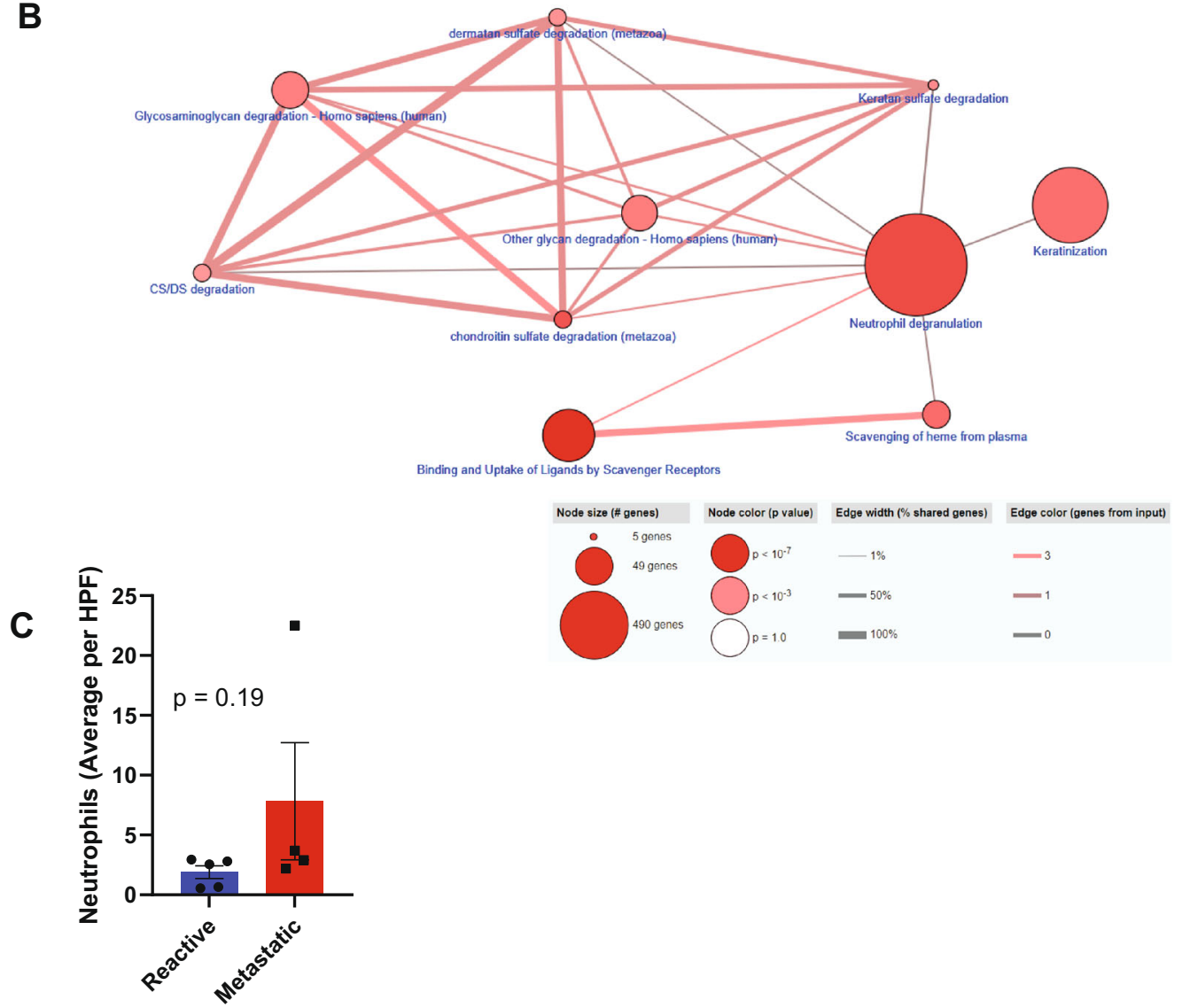

Fig. 3 The perfusate proteome reflects the pathophysiology of the axillary lymph node (ALN) from which it derives. The 57 significantly up-regulated proteins in reactive $\operatorname{ALNs}(\mathbf{A} ; n=5)$ and the 62 significantly up-regulated proteins in macrometastatic ALNs $(\mathbf{B} ; n=4)$ were subjected to pathway analysis in ConsensusPathDB. Reactive ALNs maintained immune function whilst this was lost in macrometastatic nodes, with the notable exception on 'neutrophil degranulation'. The identification of 'keratinisation' in macrometastatic nodes reflects the presence of cancer cells within the node. The other pathways identified in these nodes related to extracellular matrix (ECM) degradation. Histological neutrophil counts (haematoxylin and eosin stained ALN tissue sections) are shown in (C; graph shows mean with standard error of the mean (SEM)).

in metastatic LNs is not currently well understood. Interestingly, these data could not be obtained by stratifying TCGA(CPTAC) primary BC proteome according to ALN status, which highlights the novelty and importance of perfusate sample collection/ analysis in trying to understand metastasis.
Qualitative comparison of the perfusate proteome with the tissue proteomes of $\mathrm{LN}$-positive pancreatic ductal and prostatic adenocarcinoma showed that 438 proteins were commonly expressed. This could be technical to some extent, since it has been shown that increasing the number of sample replicates in 
A

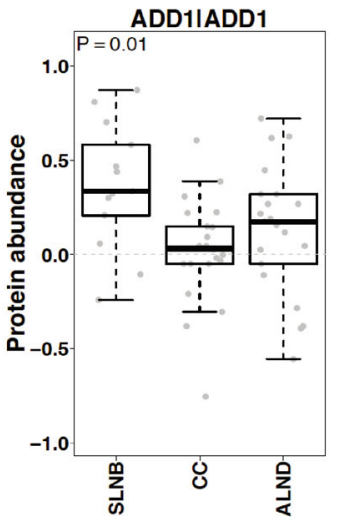

B

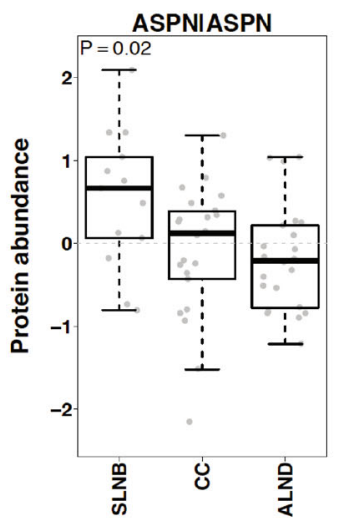

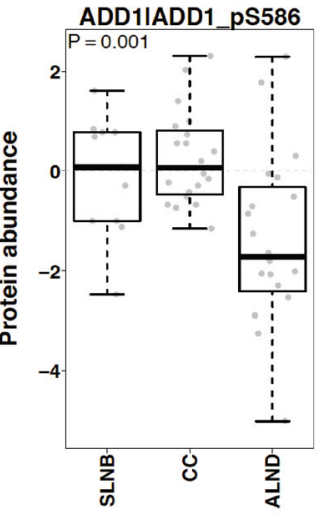
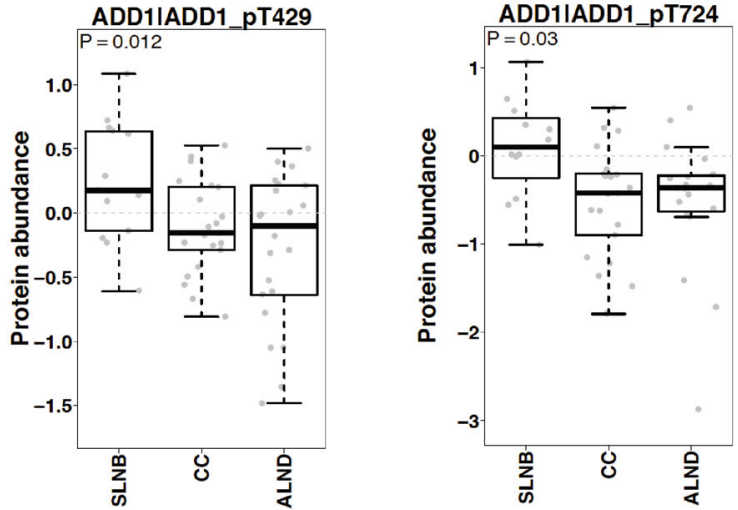

C

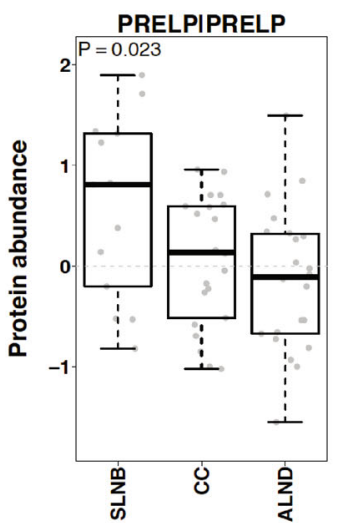

Lobo et al

Chen et al

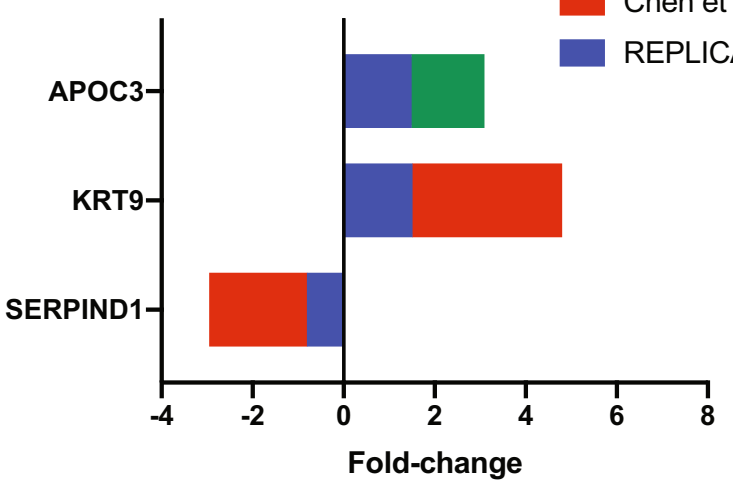

Fig. 4 Comparison of the perfusate proteome to primary breast cancer (BC) and plasma proteomes yields novel data. We stratified the TCGA(CPTAC) primary BC proteomics data into three groups according to axillary tumour burden (ATB): those who had a sentinel lymph node biopsy only (SLNB; no/low ATB); those who had a completion clearance (CC; higher burden of ATB than the SLNB group); and those who had an axillary lymph node dissection, and high ATB, at diagnosis (ALND). Of the 10 most abundant reactive proteins, ADD1 ( $p=0.01 ;$ A) and specific ADD1 phospho-isoforms; ASPN $(p=0.02 ;$ B $)$ and $\operatorname{PRELP}(p=0.023$; B) were significantly up-regulated in the SLNB group, matching our data. No metastatic proteins were found to be significantly differentially expressed. Comparison of the perfusate proteome with BC plasma proteomic studies (C) showed minimal overlap, with APOC3 and KRT9 identified as up-regulated in metastatic nodes/perfusate, and SERPIND1 as up-regulated in reactive nodes/perfusate. (Graphs show median with interquartile range).

a proteomics experiment can correct for biological diversity ${ }^{9,52,53}$. Still, if one considers that sample type and collection methods differed between the studies, and that different proteomic technologies were used, this degree of overlap suggests that recurrent biological phenomena are being detected between cancers types. The fact that 48 'core proteins' are conserved across these datasets is even more thoughtprovoking. When these were subjected to pathway analysis, the PI3K-Akt signalling pathway was the most significant one identified. This pathway is frequently dysregulated in BC, PDAC and prostate cancer, and is currently being targeted therapeutically in clinical trials ${ }^{54-59}$. Similarly, the role of various $\beta 1$ integrins in carcinogenesis is well established, particularly in terms of their interactions with collagen and the $\mathrm{ECM}^{60}$. Interestingly, the integrins $\alpha 4 \beta 1$ and $\alpha 9 \beta 1$ are known to induce lymphangiogenesis and LN metastasis; for $\alpha 4 \beta 1$, this is mediated by PI3Kalpha ${ }^{61-64}$. Key questions for future experiments will be to ascertain if these 'core proteins' are reproducibly expressed in perfusate samples harvested from other node-positive adenocarcinomas; if they can delineate nodal tumour burden; and if combinations of these proteins can be used as biomarkers of nodal spread.

Finally, 'neutrophil degranulation' was highlighted recurrently by pathway analysis as being important to LN metastasis. The role of neutrophil degranulation in cancer metastasis is gaining interest, particularly in terms of how it affects the adaptive T cell response ${ }^{65}$. Like macrophages, neutrophils can either promote or suppress immunity via cell-to-cell contact, degranulation of intracellular contents, the release/production of neutrophil extracellular traps (NETs) and/or cytokine release ${ }^{65}$. Recent evidence suggests that clusters of circulating tumour cells and neutrophils accelerate haematogenous metastasis in $\mathrm{BC}^{66}$, and that neutrophils are required for IL11-induced and FIGF-induced polyclonal BC metastasis ${ }^{67}$. Precisely how neutrophils contribute to nodal metastasis in $\mathrm{BC}$ has yet to be elucidated. We did not see statistically significant differences in absolute neutrophil numbers between reactive and metastatic perfused nodes. It will be interesting to investigate the relationship between neutrophil tissue infiltration and neutrophil degranulation in future experiments, ideally in a larger cohort of samples taken from BC patients, as well as from patients with other epithelial malignancies.

We have shown for the first time that proteomic analysis of REPLICANT perfusate ${ }^{8}$ is feasible, and reflects colonisationinduced changes in the ALN microenvironment ${ }^{6}$. Our data also suggests that these findings could be relevant to other epithelial malignancies. Future work will include validating these findings in a larger series of perfused ALNs, including micrometastatic disease. 

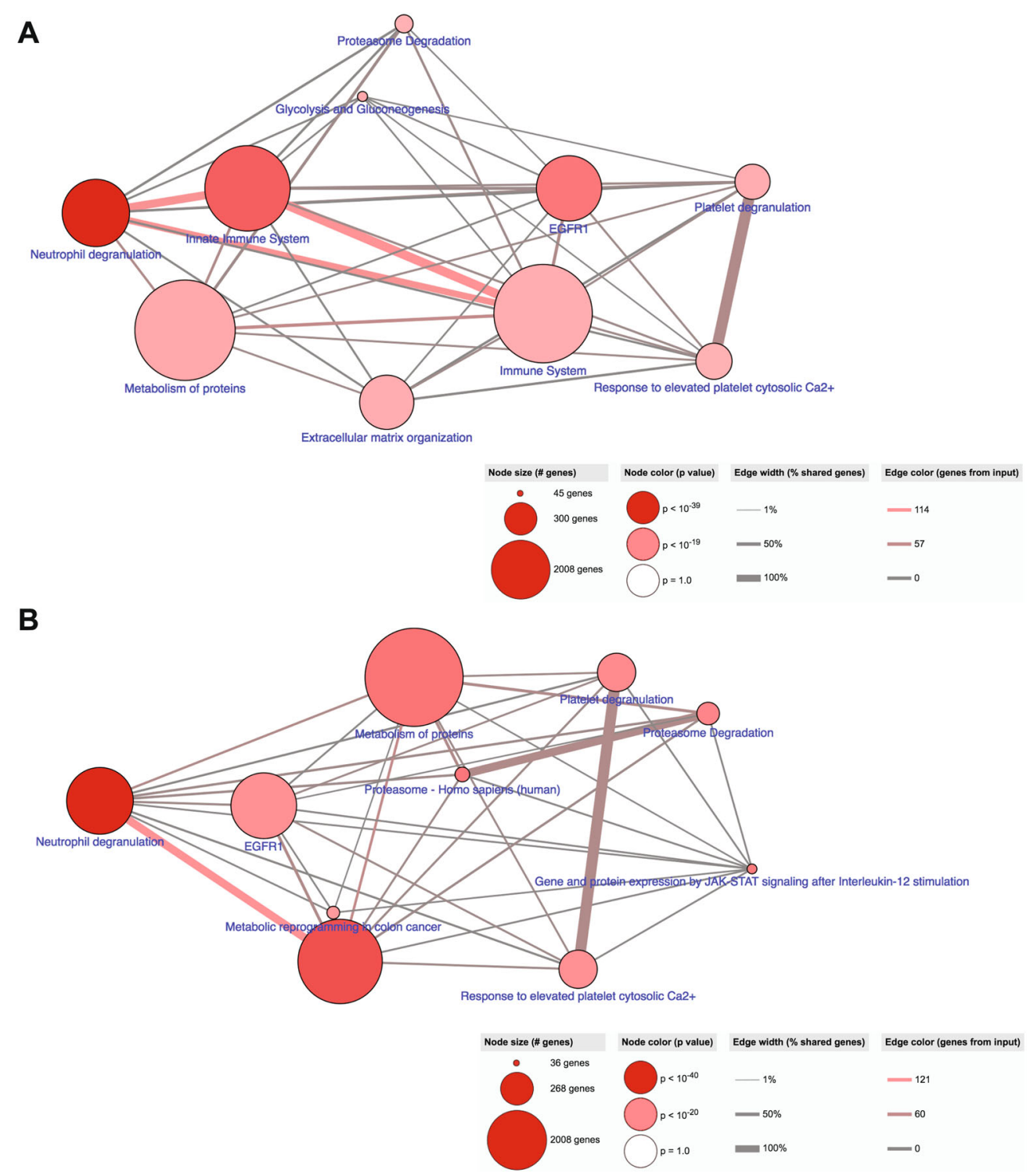

Fig. 5 Neutrophil degranulation is recurrently highlighted when the REPLICANT proteome is compared to either pancreatic or prostatic cancer. Pathway analysis of the commonly expressed proteins obtained from an exclusive comparison of the perfusate proteome to nodepositive pancreatic ductal adenocarcinoma (A; 515 commonly expressed proteins) or node-positive prostatic adenocarcinoma (B; 854 commonly expressed proteins) is shown. 'Neutrophil degranulation' was consistently identified as being important to lymph node metastasis.

\section{METHODS}

\section{Patient cohort and ALN harvest}

ALNs were harvested from $10 \mathrm{BC}$ patients and perfused ex vivo at $37^{\circ} \mathrm{C}$ as described previously (King's Health Partners (KHP) Cancer Biobank Research Ethics Committee No: 18/EE/0025) ${ }^{8}$. Informed consent was obtained from all patients prior to surgery. Four of the perfused ALNs contained macrometastases; one contained a micrometastasis; and five were reactive. A matched autologous 'baseline control' ALN, which mirrored the perfused ALN in terms of disease state, was harvested at time-point 0 from each patient. The clinico-pathological characteristics of the cohort, including representative H\&E sections, have been described previously ${ }^{8}$. Of note, none of the perfused ALNs showed any histological evidence of necrosis ${ }^{8}$.

\section{Perfusate collection}

Perfusate samples $(n=10)$ were collected from the perfusion circuit via three-way taps at the end-point of each experiment. Samples were stored at $-80^{\circ} \mathrm{C}$ prior to proteomic analysis.

\section{Multiplex Immunofluorescence (MIF)}

$4 \mu \mathrm{m}$ FFPE sections from each perfused $(n=10)$ and baseline control ALN $(n=10)$ were sequentially stained using an Opal 7-colour reagent kit (Akoya Bioscience) according to the manufacturer's instructions. The following antibodies were used: CD4 (Abcam 133616; Opal 520), CD8 (Dako, M710301; Opal 570), CD20 (Dako, M075529; Opal 540), PD-L1 (Cell Signalling, 13684; Opal 620), FoxP3 (Abcam, 20034; Opal 650), Pancytokeratin (Dako, M351501; Opal 690; on metastatic ALNs only), and CD68 (Dako, M087629; Opal 690 on reactive ALNs only). Control tissue samples were stained for each marker in parallel. Slides were imaged using the Vectra 3.0 pathology imaging system (Akoya Bioscience). Cell phenotyping and density (total number of cells $/ \mathrm{mm}^{2}$ ) was quantified over the entire tissue section (i.e. 50-600 fields per sample depending on the size of the ALN), using a custom algorithm developed in the inForm software package. Briefly, the algorithm was initially trained by machine learning on manually annotated examples. Samples were then batch processed to segment the tissue by tissue type, then to identify/ phenotype cells, and finally to quantify cell numbers or signal intensity ${ }^{68}$. 

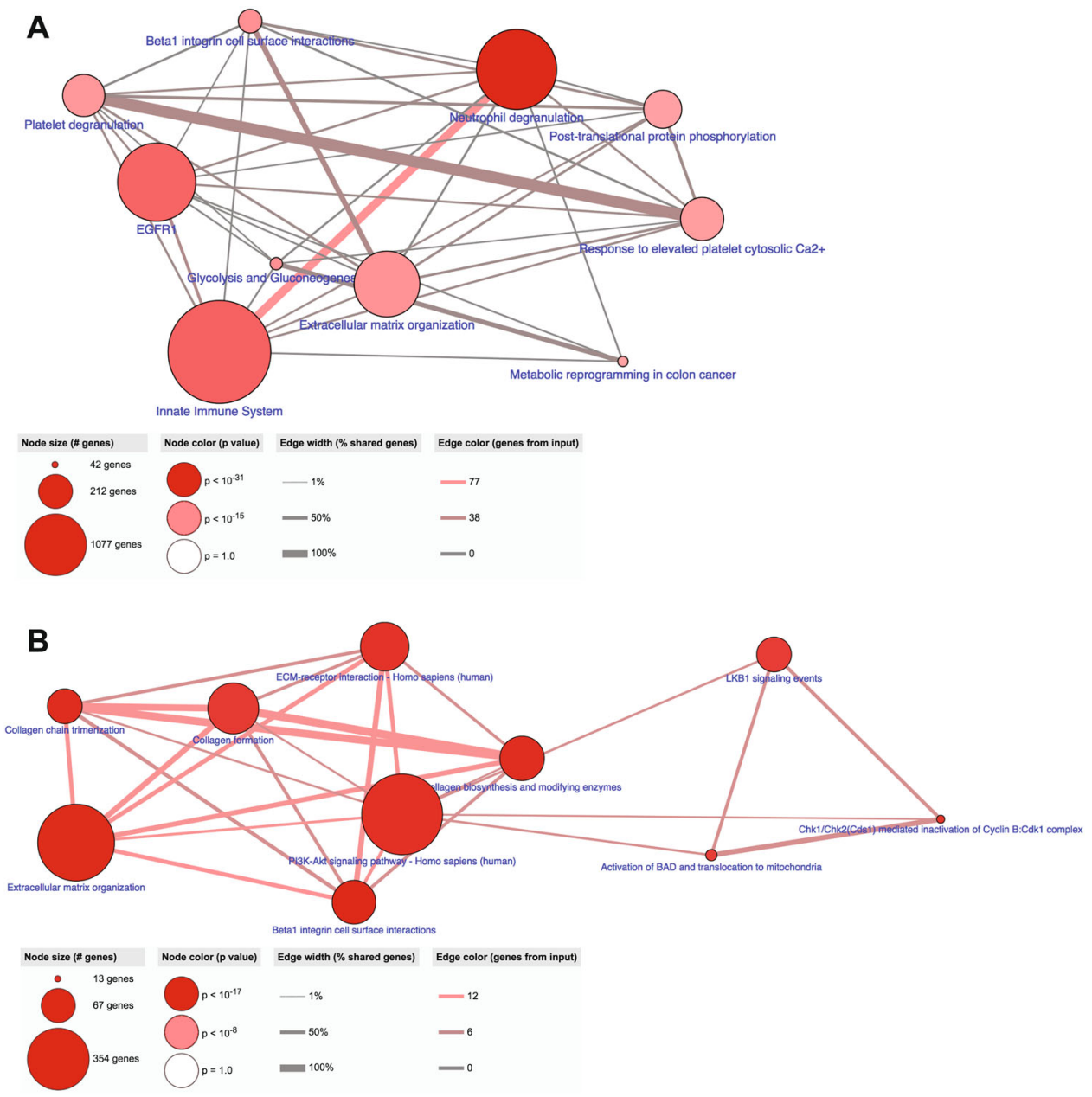

Fig. 6 Neutrophil degranulation and 48 'core proteins' are conserved in lymph node (LN) metastasis, across cancers. Concurrent comparison of the REPLICANT proteome to node-positive pancreatic and node-positive prostate adenocarcinoma identified 438 commonly expressed proteins. Pathway analysis of these 438 proteins is shown in A. 'Neutrophil degranulation' once again was identified as being important to LN metastasis. Certain protein families were also seen to recur across the three datasets ( 48 'core proteins'). Pathway analysis of these 'core proteins' is shown in B.

\section{Proteomic Analysis}

Perfusate samples were heated to $95^{\circ} \mathrm{C}$, reduced with Dithiothreitol $(50 \mathrm{mM})$ and alkylated by lodoacetamide $(100 \mathrm{mM})$. Following probe sonication, samples underwent Filter Aided Sample Preparation (FASP) in the Amicon Ultra-4 (10KDa cut-off, Millipore ${ }^{69}$. Triethylammonium bicarbonate (TEAB; $100 \mathrm{mM}$ ) was used in the buffer exchange. Peptides were recovered from the filter after an 18-h trypsin digestion (Pierce, MS grade) at $37^{\circ} \mathrm{C}$ with additional two washes of TEAB (100 mM then $\left.1 \mathrm{M}\right)$.

Following quantification (Nanodrop), $20 \mu \mathrm{g}$ of each sample was TMT labelled. The mixture was fractionated on a BEH XBridge C18 column $\left(2.1 \mathrm{~mm}\right.$ i.d. $\times 150 \mathrm{~mm}$ ) with a 35 -min gradient from $5-35 \% \mathrm{CH}_{3} \mathrm{CN} / \mathrm{NH}_{4} \mathrm{OH}$ then concatenated to 8 fractions for LC-MS/MS analysis on an Orbitrap Fusion Lumos coupled with an Ultimate 3000 RSLCnano System. Samples were loaded on a nanotrap $(100 \mu \mathrm{m}$ id $\times 2 \mathrm{~cm})($ PepMap C18, $5 \mu)$ then separated on an analytical column $(75 \mu \mathrm{m}$ id $\times 50 \mathrm{~cm})$ (PepMap C18, $2 \mu$ ) over a 90 -min gradient of $4-30.4 \% \mathrm{CH}_{3} \mathrm{CN} / 0.1 \%$ formic acid/ $120 \mathrm{~min}$ cycle time per fraction. The Orbitrap Fusion Lumos was operated in the Top Speed mode at $3 \mathrm{~s}$ per cycle and data was acquired via the MS3-SPS5 method. Raw files were processed in Proteome Discoverer 2.2 (Thermo Fisher) using the Sequest HT search engine. Spectra were searched against a reviewed Uniprot Homo sapiens database (March 2019). Peptides were validated by Percolator with q-value set at 0.01 (strict) and 0.05 (relaxed). The TMT reporter ion quantification used unique peptides only. The coisolation threshold was set at $100 \%$. Peptides with an average reported signal: noise $>3$ were used for protein quantification. Only master proteins were reported. Protein abundance was normalised by equalising the total abundance between different runs/channels, and then scaled to an average of 100 across all samples ${ }^{70}$.

\section{Neutrophil quantification}

The average number of neutrophils per high power field (HPF; 20 fields in total) was quantified in representative H\&E sections $(4 \mu \mathrm{m})$ from each anonymised ALN sample by a histopathologist (KN).

\section{The Cancer Genome Atlas (TCGA) BC proteomics analysis}

TCGA BC proteomics dataset (Clinical Proteomic Tumour Analysis Consortium (CPTAC) study) ${ }^{37-39}$ was downloaded from cBioportal (February 2019$)^{71}$. The 74 patient primary BC samples in this dataset were stratified into three groups according to the provided clinical data: those who had undergone only a sentinel lymph node biopsy ('SLNB'; $n=13$ ), and therefore had no/low burden of axillary disease; those who had gone on after a SLNB to have a completion clearance ('CC'; $n=22)$, and therefore had a higher axillary tumour burden than the SLNB group; and those who had an ALND upfront ('ALND', $n=22$ ), and therefore had a high axillary tumour burden at diagnosis. Statistical comparison of protein abundance between these three groups was performed using one-way ANOVA. Tukey HSD test was applied for post-hoc analysis. Visualisations and analysis were performed in R statistical programming environment v3.5.0. 


\section{Statistical analysis}

To assess differences in the immune composition of ALNs, a Wilcoxon test was used to compare control to perfused nodes; a Kruskal-Wallis test was used to assess regulatory $\mathrm{T}$ cell (T-regs) and lymphocyte distribution within metastatic nodes, and to compare T-reg numbers between reactive and metastatic nodes; and a two-tailed Mann-Whitney was used to quantify differences between reactive and metastatic nodes. For the proteomics analysis, the data was initially filtered to include only those proteins with a scaled normalised protein abundance $\geq 2$. A Student's $t$-test was then performed to determine differential expression between reactive and metastatic perfusate samples. A $p$-value $\leq 0.05$ was considered significant. Pathway enrichment analysis was performed with ConsensusPathDB ${ }^{31}$.

\section{Reporting summary}

Further information on experimental design is available in the Nature Research Reporting Summary linked to this paper.

\section{DATA AVAILABILITY}

The mass-spectrometry based proteomics data generated during the study, are publicly available in the PRIDE repository: https://identifiers.org/pride.project: PXD022722 $2^{70}$. The multiplex immunofluorescence data generated during this study, are available in the figshare repository: https://doi.org/10.6084/m9.figshare.13522442 ${ }^{68}$. The TCGA data analysed during the study, are available in the cBioPortal for Cancer Genomics: https://identifiers.org/cbioportal:brca_tcga ${ }^{71}$. All other data supporting the findings of this study, are available as part of the supplementary files that accompany the article.

Received: 20 July 2020; Accepted: 20 January 2021; Published online: 05 March 2021

\section{REFERENCES}

1. Naidoo, K. \& Pinder, S. E. Micro- and macro-metastasis in the axillary lymph node: a review. Surgeon 15, 76-82 (2017).

2. de Boer, M. et al. Micrometastases or isolated tumor cells and the outcome of breast cancer. N. Engl. J. Med. 361, 653-663 (2009).

3. de Boer, M., van Dijck, J. A., Bult, P., Borm, G. F. \& Tjan-Heijnen, V. C. Breast cancer prognosis and occult lymph node metastases, isolated tumor cells, and micrometastases. J. Natl Cancer Inst. 102, 410-425 (2010).

4. Giuliano, A. E. et al. Locoregional recurrence after sentinel lymph node dissection with or without axillary dissection in patients with sentinel lymph node metastases: the American College of Surgeons Oncology Group Z0011 randomized trial. Ann. Surg. 252, 426-432 (2010).

5. Ahmed, M. \& Douek, M. Life beyond Z11. Breast 22, 1226-1227 (2013).

6. Fidler, I. J. The pathogenesis of cancer metastasis: the 'seed and soil' hypothesis revisited. Nat. Rev. Cancer 3, 453-458 (2003).

7. NICE. Early and Locally Advanced Breast Cancer: Diagnosis And Management. https://www.nice.org.uk/guidance/ng101 (2018).

8. Barrow-McGee, R. et al. Real-time ex vivo perfusion of human lymph nodes invaded by cancer (REPLICANT): a feasibility study. J. Pathol. https://doi.org/ 10.1002/path.5367 (2019).

9. Orton, D. J. \& Doucette, A. A. Proteomic workflows for biomarker identification using mass spectrometry-technical and statistical considerations during initial discovery. Proteomes 1, 109-127 (2013).

10. Barry, P. et al. The spatiotemporal evolution of lymph node spread in early breast cancer. Clin. Cancer Res. 24, 4763-4770 (2018).

11. Merker, J. D. et al. Circulating tumor DNA analysis in patients with cancer: American society of clinical oncology and college of american pathologists joint review. J. Clin. Oncol. 36, 1631-1641 (2018).

12. Li, J., Zhang, Z., Rosenzweig, J., Wang, Y. Y. \& Chan, D. W. Proteomics and bioinformatics approaches for identification of serum biomarkers to detect breast cancer. Clin. Chem. 48, 1296-1304 (2002).

13. Nakagawa, T. et al. Proteomic profiling of primary breast cancer predicts axillary lymph node metastasis. Cancer Res. 66, 11825-11830 (2006).

14. Pietrowska, M. et al. Mass spectrometry-based serum proteome pattern analysis in molecular diagnostics of early stage breast cancer. J. Transl. Med. 7, 60 (2009).

15. Wang, L. et al. Primary study of lymph node metastasis-related serum biomarkers in breast cancer. Anat. Rec. 294, 1818-1824 (2011).

16. Lobo, M. D. et al. Label-free proteome analysis of plasma from patients with breast cancer: stage-specific protein expression. Front. Oncol. 7, 14 (2017).
17. Chen, L. et al. Label-free quantitative proteomic screening of candidate plasma biomarkers for the prognosis of breast cancer with different lymph node statuses. Proteom. Clin. Appl. 12, e1700117 (2018).

18. Thongwatchara, P. et al. Differential protein expression in primary breast cancer and matched axillary node metastasis. Oncol. Rep. 26, 185-191 (2011).

19. Li, J. et al. Omics-based profiling of carcinoma of the breast and matched regional lymph node metastasis. Proteomics 8, 5038-5052 (2008).

20. Milioli, H. H. et al. Comparative proteomics of primary breast carcinomas and lymph node metastases outlining markers of tumor invasion. Cancer Genomics Proteom. 12, 89-101 (2015).

21. Zeng, L. et al. Identification of nucleobindin-2 as a potential biomarker for breast cancer metastasis using iTRAQ-based quantitative proteomic analysis. J. Cancer $\mathbf{8}$, 3062-3069 (2017).

22. Pozniak, Y. et al. System-wide clinical proteomics of breast cancer reveals global remodeling of tissue homeostasis. Cell Syst. 2, 172-184 (2016).

23. Zeng, L. et al. Prognostic value of biomarkers EpCAM and alphaB-crystallin associated with lymphatic metastasis in breast cancer by ITRAQ analysis. BMC Cancer 19, 831 (2019).

24. Broggi, M. A. S. et al. Tumor-associated factors are enriched in lymphatic exudate compared to plasma in metastatic melanoma patients. J. Exp. Med. 216, 1091-1107 (2019).

25. Clement, C. C. et al. Protein expression profiles of human lymph and plasma mapped by 2D-DIGE and 1D SDS-PAGE coupled with nanoLC-ESI-MS/MS bottomup proteomics. J. Proteom. 78, 172-187 (2013).

26. Dzieciatkowska, M. et al. Lymph is not a plasma ultrafiltrate: a proteomic analysis of injured patients. Shock 42, 485-498 (2014)

27. Leak, L. V. et al. Proteomic analysis of lymph. Proteomics 4, 753-765 (2004).

28. Hendry, S. et al. Assessing tumor-infiltrating lymphocytes in solid tumors: a practical review for pathologists and proposal for a standardized method from the international immunooncology biomarkers working group: part 1: assessing the host immune response, TILs in invasive breast carcinoma and ductal carcinoma in situ, metastatic tumor deposits and areas for further research. Adv. Anat. Pathol. 24, 235-251 (2017).

29. Togashi, Y., Shitara, K. \& Nishikawa, H. Regulatory $T$ cells in cancer immunosuppression-implications for anticancer therapy. Nat. Rev. Clin. Oncol. 16, 356-371 (2019).

30. Sun, C., Mezzadra, R. \& Schumacher, T. N. Regulation and function of the PD-L1 checkpoint. Immunity 48, 434-452 (2018).

31. Kamburov, A. et al. ConsensusPathDB: toward a more complete picture of cell biology. Nucleic Acids Res. 39, D712-D717 (2011).

32. Wiig, H. \& Swartz, M. A. Interstitial fluid and lymph formation and transport: physiological regulation and roles in inflammation and cancer. Physiol. Rev. 92, 1005-1060 (2012)

33. Johansson, A., Hamzah, J. \& Ganss, R. More than a scaffold: stromal modulation of tumor immunity. Biochim. Biophys. Acta 1865, 3-13 (2016).

34. Gretz, J. E., Kaldjian, E. P., Anderson, A. O. \& Shaw, S. Sophisticated strategies for information encounter in the lymph node: the reticular network as a conduit of soluble information and a highway for cell traffic. J. Immunol. 157, 495-499 (1996).

35. Fletcher, A. L., Acton, S. E. \& Knoblich, K. Lymph node fibroblastic reticular cells in health and disease. Nat. Rev. Immunol. 15, 350-361 (2015).

36. Martinez, V. G. et al. Fibroblastic reticular cells control conduit matrix deposition during lymph node expansion. Cell Rep. 29, 2810-2822 e2815 (2019).

37. Edwards, N. J. et al. The CPTAC data portal: a resource for cancer proteomics research. J. Proteome Res. 14, 2707-2713 (2015).

38. Ellis, M. J. et al. Connecting genomic alterations to cancer biology with proteomics: the $\mathrm{NCl}$ Clinical Proteomic Tumor Analysis Consortium. Cancer Discov. 3, 1108-1112 (2013).

39. Mertins, P. et al. Proteogenomics connects somatic mutations to signalling in breast cancer. Nature 534, 55-62 (2016).

40. Brierley J. D., G. M. a. W. C. TNM Classification of Malignant Tumours, 8th edn. (Wiley-Black, 2016).

41. Naidoo, K. et al. Proteome of formalin-fixed paraffin-embedded pancreatic ductal adenocarcinoma and lymph node metastases. J. Pathol. 226, 756-763 (2012).

42. Muller, A. K. et al. Proteomic characterization of prostate cancer to distinguish nonmetastasizing and metastasizing primary tumors and lymph node metastases. Neoplasia 20, 140-151 (2018).

43. Buisseret, L. et al. Tumor-infiltrating lymphocyte composition, organization and PD-1/ PD-L1 expression are linked in breast cancer. Oncoimmunology 6, e1257452 (2017).

44. Noske, A. et al. Relevance of tumour-infiltrating lymphocytes, PD-1 and PD-L1 in patients with high-risk, nodal-metastasised breast cancer of the German Adjuvant Intergroup Node-positive study. Eur. J. Cancer 114, 76-88 (2019).

45. Yeong, J. et al. Multiplex immunohistochemistry/immunofluorescence (mIHC/IF) for PD-L1 testing in triple-negative breast cancer: a translational assay compared 
with conventional IHC. J. Clin. Pathol. https://doi.org/10.1136/jclinpath-2019206252 (2020).

46. Bensch, F. et al. (89)Zr-atezolizumab imaging as a non-invasive approach to assess clinical response to PD-L1 blockade in cancer. Nat. Med. 24, 1852-1858 (2018).

47. Szekely, B. et al. Immunological differences between primary and metastatic breast cancer. Ann. Oncol. 29, 2232-2239 (2018).

48. Schmid, P. et al. Atezolizumab and Nab-paclitaxel in advanced triple-negative breast cancer. N. Engl. J. Med. 379, 2108-2121 (2018).

49. Lu, P., Weaver, V. M. \& Werb, Z. The extracellular matrix: a dynamic niche in cancer progression. J. Cell Biol. 196, 395-406 (2012).

50. Psaila, B. \& Lyden, D. The metastatic niche: adapting the foreign soil. Nat. Rev. Cancer 9, 285-293 (2009).

51. Paolillo, M. \& Schinelli, S. Extracellular matrix alterations in metastatic processes. Int. J. Mol. Sci. 20, https://doi.org/10.3390/ijms20194947 (2019).

52. Ghaemmaghami, S. et al. Global analysis of protein expression in yeast. Nature 425, 737-741 (2003).

53. Liu, H., Sadygov, R. G. \& Yates, J. R. 3rd A model for random sampling and estimation of relative protein abundance in shotgun proteomics. Anal. Chem. 76, 4193-4201 (2004).

54. Cancer Genome Atlas, N. Comprehensive molecular portraits of human breast tumours. Nature 490, 61-70 (2012).

55. Armenia, J. et al. The long tail of oncogenic drivers in prostate cancer. Nat. Genet 50, 645-651 (2018).

56. Conway, J. R., Herrmann, D., Evans, T. J., Morton, J. P. \& Timpson, P. Combating pancreatic cancer with $\mathrm{PI} 3 \mathrm{~K}$ pathway inhibitors in the era of personalised medicine. Gut 68, 742-758 (2019).

57. Liu, P., Cheng, H., Roberts, T. M. \& Zhao, J. J. Targeting the phosphoinositide 3kinase pathway in cancer. Nat. Rev. Drug Discov. 8, 627-644 (2009).

58. Sarker, D., Reid, A. H., Yap, T. A. \& de Bono, J. S. Targeting the PI3K/AKT pathway for the treatment of prostate cancer. Clin. Cancer Res. 15, 4799-4805 (2009).

59. Turner, N. C., Neven, P., Loibl, S. \& Andre, F. Advances in the treatment of advanced oestrogen-receptor-positive breast cancer. Lancet 389, 2403-2414 (2017).

60. Raab-Westphal, S., Marshall, J. F. \& Goodman, S. L. Integrins as therapeutic targets: successes and cancers. Cancers 9, https://doi.org/10.3390/cancers9090110 (2017).

61. Garmy-Susini, B. et al. PI3Kalpha activates integrin alpha4beta1 to establish a metastatic niche in lymph nodes. Proc. Natl Acad. Sci. USA 110, 9042-9047 (2013).

62. Garmy-Susini, B. et al. Integrin alpha4beta1 signaling is required for lymphangiogenesis and tumor metastasis. Cancer Res. 70, 3042-3051 (2010).

63. Vlahakis, N. E., Young, B. A., Atakilit, A. \& Sheppard, D. The lymphangiogenic vascular endothelial growth factors VEGF-C and $-D$ are ligands for the integrin alpha9beta1. J. Biol. Chem. 280, 4544-4552 (2005).

64. Hoye, A. M., Couchman, J. R., Wewer, U. M., Fukami, K. \& Yoneda, A. The newcomer in the integrin family: integrin alpha9 in biology and cancer. Adv. Biol. Regul. 52, 326-339 (2012).

65. Minns, D., Smith, K. J. \& Findlay, E. G. Orchestration of adaptive T cell responses by neutrophil granule contents. Mediat. Inflamm. 2019, 8968943, (2019).

66. Szczerba, B. M. et al. Neutrophils escort circulating tumour cells to enable cell cycle progression. Nature 566, 553-557 (2019).

67. Janiszewska, M. et al. Subclonal cooperation drives metastasis by modulating local and systemic immune microenvironments. Nat. Cell Biol. 21, 879-888 (2019).

68. Stevenson, J. et al. Multiplex immunofluorescence data and metadata supporting the article: proteomics of REPLICANT perfusate detects changes in the metastatic lymph node microenvironment. figshare https://doi.org/10.6084/m9.figshare.13522442 (2021).

69. Wisniewski, J. R., Zougman, A., Nagaraj, N. \& Mann, M. Universal sample preparation method for proteome analysis. Nat. Methods 6, 359-362 (2009).

70. Stevenson, J. et al. Proteomics of REPLICANT perfusate detects changes in the metastatic lymph node microenvironment. PRIDE Archive https://identifiers.org/ pride.project:PXD022722 (2021)

71. TCGA Breast Invasive Carcinoma. cBioPortal for Cancer Genomics, https:// identifiers.org/cbioportal:brca_tcga (2016).

\section{ACKNOWLEDGEMENTS}

The authors would like to thank the patients for consenting for use of their tissue to develop this model, and the surgeons who assisted with this. This work was funded by Cancer Research UK (Pioneer Award CRC598X). We thank Breast Cancer Now for funding this work as part of Programme Funding to the Breast Cancer Now Toby Robins Research Centre. This work was funded by a Clinical Lectureship at The Institute of Cancer Research from the National Institute of Health Research (NIHR) to Dr Kalnisha Naidoo. This study represents independent research supported by the NIHR Biomedical Research Centre (BRC) at the Royal Marsden NHS Foundation Trust and The Institute of Cancer Research, London. The views expressed are those of the authors and not necessarily those of the NIHR or the Department of Health and Social Care.

\section{AUTHOR CONTRIBUTIONS}

K.N. conceived the study; J.S., R.B.M. and K.N. designed the experiments, with advice from A.N.J.T. and S.E.P.; R.B.M., K.N., A.N.J.T. and S.E.P. applied for ethical study approval; J.O., N.W., K.N., S.E.P. and C.G. oversaw the collection, use and storage of human tissue and perfusate samples through the King's Health Partners Biobank; R.B.M., J.P. and K.N. perfused the ALNs used in the study, and collected the perfusate samples for analysis; L.Y., A.P. and J.C. prepared the perfusate samples for, and performed, LC-MS/MS; K.N. statistically analysed the proteomics data with input from L.Y. and J.C.; N.W. and J.O. cut whole sections from tissue samples which D.M. stained and quantified for MIF with histopathological input from K.N.; K.N. performed the statistical analysis of MIF data with input from D.M.; S.H. performed the bioinformatics analysis of TCGA(CPTAC) data with input from R.N. and K.N.; J.S., R.B.M. and K.N. wrote the manuscript, with critical input from all other authors.

\section{COMPETING INTERESTS}

The authors declare no competing interests.

\section{ADDITIONAL INFORMATION}

Supplementary information The online version contains supplementary materia available at https://doi.org/10.1038/s41523-021-00227-7.

Correspondence and requests for materials should be addressed to K.N.

Reprints and permission information is available at http://www.nature.com/ reprints

Publisher's note Springer Nature remains neutral with regard to jurisdictional claims in published maps and institutional affiliations.

\begin{abstract}
(i) Open Access This article is licensed under a Creative Common Attribution 4.0 International License, which permits use, sharing, adaptation, distribution and reproduction in any medium or format, as long as you give appropriate credit to the original author(s) and the source, provide a link to the Creative Commons license, and indicate if changes were made. The images or other third party material in this article are included in the article's Creative Commons license, unless indicated otherwise in a credit line to the material. If material is not included in the article's Creative Commons license and your intended use is not permitted by statutory regulation or exceeds the permitted use, you will need to obtain permission directly from the copyright holder. To view a copy of this license, visit http://creativecommons. org/licenses/by/4.0/.
\end{abstract}

(c) The Author(s) 2021 